\title{
Video Article \\ A Positioning Device for the Placement of Mice During Intranasal siRNA Delivery to the Central Nervous System
}

\author{
Irfan Ullah ${ }^{1,2}$, Kunho Chung ${ }^{1}$, Jagadish Beloor ${ }^{2}$, Sang-Kyung Lee ${ }^{1}$, Priti Kumar $^{2}$ \\ ${ }^{1}$ Department of Bioengineering and Institute of Nanoscience and Technology, Hanyang University \\ ${ }^{2}$ Department of Internal Medicine, Section of Infectious Diseases, Yale University School of Medicine
}

Correspondence to: Sang-Kyung Lee at sangkyunglee@hanyang.ac.kr, Priti Kumar at priti.kumar@yale.edu

URL: https://www.jove.com/video/59201

DOI: doi:10.3791/59201

Keywords: Immunology and Infection, Issue 150, intranasal administration, brain delivery, siRNA, RVG9R peptide, mouse positioning device, noseto-brain

Date Published: 8/15/2019

Citation: Ullah, I., Chung, K., Beloor, J., Lee, S.K., Kumar, P. A Positioning Device for the Placement of Mice During Intranasal siRNA Delivery to the Central Nervous System. J. Vis. Exp. (150), e59201, doi:10.3791/59201 (2019).

\section{Abstract}

Intranasal (IN) drug delivery to the brain has emerged as a promising method to bypass the blood-brain barrier (BBB) for the delivery of drugs into the central nervous system (CNS). Recent studies demonstrate the use of a peptide, RVG9R, incorporating the minimal receptor-binding domain of the Rabies virus glycoprotein, in eliciting the delivery of siRNA into neurons in the brain. In this protocol, the peptide-siRNA formulation is delivered intranasally with a pipette in the dominant hand, while the anesthetized mouse is restrained by the scruff with the nondominant hand in a "head down-and-forward position" to avoid drainage into the lung and stomach upon inhalation. This precise gripping of mice can be learned but is not easy and requires practice and skill to result in effective CNS uptake. Furthermore, the process is long-drawn, requiring about 45 min for the administration of a total volume of $\sim 20-30 \mu \mathrm{L}$ of solution in 1-2 $\mu \mathrm{L}$ droplet volumes per inhalation, with 3-4 min rest periods between each inhalation. The objective of this study is to disclose a mouse positioning device that enables the appropriate placement of mice for efficient IN administration of the peptide-siRNA formulation. Multiple features are incorporated into the design of the device, such as four or eight positioning chairs with adjustable height and tilt to restrain anesthetized mice in the head down-and-forward position, enabling easy visualization of the mice's nares and a built-in heating pad to maintain the mice's body temperatures during the procedure. Importantly, the ability to treat four or eight mice simultaneously with RVG9R-siRNA complexes in this manner enables studies on a much quicker time scale, for the testing of an IN therapeutic siRNA approach. In conclusion, this device allows for appropriate and controlled mouse head positioning for IN application of RVG9R-siRNA and other therapeutic molecules, such as nanoparticles or antibodies, for CNS delivery.

\section{Video Link}

The video component of this article can be found at https://www.jove.com/video/59201/

\section{Introduction}

The BBB prevents systemically administered molecules of $>400-600$ Da from entering the brain, posing a significant challenge to the delivery of therapeutic biomolecules for diseases affecting the CNS and brain ${ }^{1}$. Direct drug delivery to the brain can be achieved by stereotactic injection; however, this requires surgical expertise and is highly restricted in delivery to areas proximal to the injection site, making it unsuitable for routine clinical use ${ }^{2}$. IN delivery to the brain can also result in direct brain delivery by bypassing the BBB, allowing for the direct and rapid transfer of a variety of substances to the brain ${ }^{3,4}$. This transfer is thought to occur by transport mechanisms via the olfactory and trigeminal nerves that connect the nasal passage to the brain, the cerebrospinal fluid, and the lymphatic systems ${ }^{5}$. As the direct nose-to-brain route does not involve peripheral organs and tissues, it substantially reduces systemic side effects and improves potency. IN administration is a promising noninvasive alternative to both local and systemic routes for brain delivery of therapeutic agents and may represent a powerful approach to combat neurological disorders, including Alzheimer's disease, Parkinson's disease, and brain cancer, and is being explored in several clinical trials $^{6,7,8}$.

Several experimental factors, such as volume and method of inoculation, as well as formulation $\mathrm{pH}$, strongly influence drug delivery to the CNS via the nose-to-brain pathway 9 . In studies with mice, the success of IN drug delivery strongly depends on proper head positioning, which is critical for efficient brain deposition and to avoid drug drainage into the external environment or the airways. Notably, the majority of rodent studies employ a head-back position (supine) with a $70^{\circ}-90^{\circ}$ tilt for drug delivery to the olfactory epithelium, even though head positioning at $0^{\circ}$ may favor drainage into the trachea ${ }^{9}$. IN delivery of drugs in mice that are awake results in reduced brain deposition compared to any application in the supine position, mostly due to scientists' inability to hold mice in the desired position for longer periods of time. Moreover, the upsidedown position required by the skin grip method employed for awake mice results in drug deposition predominantly in the trigeminal nerve and the olfactory bulb, as well as peripheral organs, such as kidneys and lungs, within 30 min postinoculation ${ }^{10}$. The most appropriate body position for the delivery of therapeutics through the olfactory or trigeminal nerves in larger animals such as nonhuman primates in clinical studies appears to be the head down-and-forward position (i.e., the so-called "praying to Mecca position") ${ }^{11}$. However, this position has not been well-studied in the mouse model, and the supine position is more widely used in rodent studies. 
Previously, we have shown that the RVG9R, a peptide designed based on the minimal receptor binding domain of the Rabies virus, displays tropism to cells expressing nicotinic acetylcholine receptor subunits like neurons and macrophages and mediates the intracellular delivery of siRNA by a mechanism involving receptor engagement and temporary plasma membrane delocalization at the site of receptor aggregation ${ }^{12,13}$. Importantly, systemic intravenous administration of RVG9R-siRNA complexes enables the transvascular delivery of siRNA into the CNS ${ }^{14}$. However, the systemic route dilutes the amount of siRNA delivered to the CNS, and recent data demonstrate that the IN administration of RVG9R:siRNA complexes to mice positioned in the head down-and-forward position elicits wide-spread target gene knockdown in multiple regions of the brain ${ }^{15}$. Importantly, this level of knockdown was achieved with as little as $13.5 \mu \mathrm{g}$ of siRNA administered over a four-dose, 2 day regimen while the IV route requires a $\sim 5$ times higher dose per injection to achieve comparable knockdown. The only shortcoming of the IN approach is that it is an arduous procedure, requiring the use of both hands during the administration of the solution while continuously gripping the mice alternatively in the head down-and-forward and in relaxed positions between each inhalation for a considerable long treatment period (a 30-45 min procedure for the effective uptake of a $\sim 20-30 \mu \mathrm{L}$ volume per mouse). Using the mouse positioning device presented here enables the proper placement of mice with little physical duress to the animals and the personnel performing the protocol, as well as the treatment of multiple cohorts of mice within a reasonable period of time, enabling an in-depth study on using siRNAs as a therapeutic for West Nile encephalitis in mice at late stages of the disease ${ }^{15}$.

\section{Protocol}

All experiments were performed in compliance with guidelines and protocols approved by the Animal Care and Use Committee of the Hanyang University. In this protocol, 6-week-old Balb/c mice weighing 20-25 g were used ( $n=3$ per group). The animals were housed in a pathogen-free facility with $12 \mathrm{~h}$ light/dark cycles at a controlled temperature and humidity level with free access to water and food.

\section{Device assembly}

1. Assemble the individual parts of the positioning device as shown in Figure $\mathbf{1 A}$

NOTE: The device is supplied in individual parts that can be easily assembled and disassembled.

\section{Material setup}

NOTE: The IN administration of liquefied drugs requires the following pretreatment steps.

1. Prepare the required materials, including the positioning device, a $10 \mu \mathrm{L}$ micropipette, $10 \mu \mathrm{L}$ micropipette tips, a treatment solution (e.g., RVG9R-siRNA complex), a timer clock, and $70 \%$ ethanol (refer to Figure 2A).

2. Plug the power code to turn on the device heating system at least $15-20$ min prior to the experiment. The optimal temperature for animal experiments is automatically maintained at $\sim 37^{\circ} \mathrm{C}$.

3. Prepare the anesthesia ketamine:xylazine:phosphate-buffered saline (PBS) at a ratio of 1:0.5:8.5 and anesthetize each mouse with a single intraperitoneal (i.p.) injection in a final volume of $200 \mu \mathrm{L}$ per $20 \mathrm{~g}$ mouse.

NOTE: Please note that any optimized drug for ensuring the anesthesia condition for a period of 30-45 min can be used.

\section{Mouse positioning}

1. Assess the level of anesthesia by pedal reflex (firm toe pinch) to maintain the surgical plane.

2. Place the positioning device at an appropriate distance and height so as to provide for convenient access to all required reagents

3. Once the mice are properly anesthetized, gently lift each mouse by the scruff of their neck with thumb and pointer finger and place it on the designated chair.

4. Proper positioning of the mouse on the chair is very important at this stage. Lay the mouse's back parallel to the back support of the chair, and at $90^{\circ}$ to the chair seat. Lift the hands off the mouse and let the animal lie naturally in the head down-and-forward position, without pushing or pressing, as shown in Figure 2B. Make sure the mouse's forelimbs provide natural support while the animal is in this relaxed position, without any discomfort to the mouse.

5. Once the mice are properly positioned, strap the mice in with the chair belt, and immediately start the IN inoculation.

\section{Intranasal delivery}

NOTE: This protocol describes the IN delivery of RVG9R:siRNA complexes using the mouse positioning device. Using this protocol, a maximum of $20-30 \mu \mathrm{L}$ of solution can be easily administered to each mouse in $2 \mu \mathrm{L}$ drops. This volume is lower than that of the mouse's nasal cavity, which is $0.032 \mathrm{~cm}^{3}$, so the procedure does not result in lethal nostril obstruction or asphyxiation. This protocol allows for the simultaneous inoculation of at least four mice and a maximum of eight mice per device, resulting in time optimization and reduced variability.

1. Take the $10 \mu \mathrm{L}$ micropipette and adjust it to $2 \mu \mathrm{L}$.

2. Load the micropipette with $2 \mu \mathrm{L}$ of RVG9R:siRNA complex solution (e.g., $5.2 \mu \mathrm{g}$ of siCy5 complexed with $104 \mu \mathrm{g}$ of RVG9R for the delivery experiment, and $13.5 \mu \mathrm{g}$ of siSOD1 complexed with $270 \mu \mathrm{g}$ of RVG9R for the silencing experiment, in a final volume of $25 \mu \mathrm{L}$ of PBS containing $5 \%$ glucose.

3. While holding the pipette in the dominant hand, adjust position by placing an elbow on the bench top. Support the hand holding the pipette with the other hand to avoid uncontrolled movements while administering.

4. Place a $\sim 2 \mu \mathrm{L}$ drop very close to one nostril so that the mouse can directly inhale the droplet (refer to Figure 2B). If a tiny drop is not easily formed, then replace the pipette tip with a new one and repeat the operation. Use the stopwatch to verify the time interval between inoculations. 
5. This device allows for the treatment of at least four mice at a time. If another group of mice is required to repeat the step 4.4 , set another bar with four chairs above or below the first bar with four chairs to accommodate up to eight mice at a time on the device.

6. Repeat step 4.4 with the other nostril after 3-4 min from the first inoculation. This time interval is sufficient for the mouse to complete the inhalation of the first dose and restore normal breathing. If a drop of the inhaled solution is dislodged due to accidental snorting out from the mouse's naris, reinoculate an additional $2 \mu \mathrm{L}$ of solution.

7. Repeat the entire process with alternate nostrils until the $20-30 \mu \mathrm{L}$ dose is finished. It will take $\sim 30-45$ min to complete the entire inoculation procedure.

8. Put wet ointment on the mice's eyes before returning the mice back to their designated cages.

NOTE: Do not leave the mice unattended until they have regained sufficient consciousness to maintain sternal recumbency.

\section{Data analysis}

1. To confirm the brain deposition of Fluor-labeled RVG9R, anesthetize the mice (as described in step 2.3) and isolate the brain, as well as other peripheral organs, such as lung, liver, spleen, and kidney. Place each organ on the ImageStation and visualize fluorescence using ImageStation.

2. To visualize fluorescence due to the Cy5-label, prepare $20 \mu \mathrm{m}$-thick cryosections, costained with Hoechst 33342 , and perform full-brain scanning with a confocal microscope.

3. To assess gene silencing, extract RNA from various brain regions, such as the olfactory bulb, the cortex, the hippocampus, the thalamus, the hypothalamus, the cerebellum, and the brain stem, with an RNA purification kit, reverse-transcribed into cDNA using a cDNA synthesis kit, and perform $\mathrm{qPCR}$ with primer pairs as described previously ${ }^{15}$.

\section{Representative Results}

Head position during IN administration is a major influence on the efficiency of drug delivery to the brain. Here we described the head down-andforward position using a mouse positioning device for the IN administration of a brain-targeting peptide-siRNA formulation for the delivery of the mixture to the mouse's CNS. To verify delivery through the IN route, we used the RVG9R peptide, previously shown to efficiently bind to neuronal cells both in vitro and in vivo ${ }^{14,16}$.

We first tested nose-to-brain delivery of the RVG9R peptide labeled with Alexa Fluor 488 (RVG9R-A ${ }^{488}$ ) by using the mouse positioning device described here. At $48 \mathrm{~h}$ postinoculation, various organs, including the brain, the sinus, lungs, the liver, the spleen, and kidneys were excised to measure tissue-associated $A^{488}$ fluorescence. $A^{488}$ alone, or a control peptide (RVM9R-A $\left.{ }^{488}\right)^{14}$ that does not bind $n A c h R$, were used as negative controls. As expected, neither $A^{488}$ nor RVM9R-A ${ }^{488}$ were detected in any of the organs at $48 \mathrm{~h}$ postinoculation (Figure 3A, left). On the other hand, a strong fluorescent $A^{488}$ signal was detected exclusively in the brains of the RVG9R-inoculated group. In addition, we compared this mouse placement position to the supine position method that has been used previously ${ }^{17}$, as well as to the awake method, for efficacy. We inoculated a fixed amount of RVG9R-A ${ }^{488}(100 \mu \mathrm{g})$ and assayed biodistribution at $48 \mathrm{~h}$ postinoculation. The results indicated that positioning the mice head down-and-forward improved the penetrance and deposition of RVG9R-A ${ }^{488}$ throughout the brain tissue (Figure $3 A$, right). In contrast, the animals inoculated in a supine position resulted in the delivery of RVG9R-A ${ }^{488}$ to the brain but not as strong as seen with the positioning device method. To further confirm siRNA delivery to the brain, we performed full-brain scanning after a single IN administration of RVG9R complexed to $400 \mathrm{pmol}(5.2 \mu \mathrm{g})$ of Cy5-labeled siRNA. In contrast to PBS or RVM9R, complexing with RVG9R resulted in a strong accumulation of siRNA in major brain regions, including the olfactory bulb, the cortex, the hippocampus, the thalamus, the hypothalamus, the midbrain, and the cerebellum (Figure 3B).

Finally, we employed RT-qPCR analysis to verify the intracellular delivery of functional siRNA targeting the superoxide dismutase-1 (SOD1) gene. Mice were inoculated intranasally for 3 times on consecutive days with RVG9R:siSOD1 (13.2 $\mu \mathrm{g}$ of siRNA), and the SOD1 mRNA expression was analyzed $24 \mathrm{~h}$ after the last inoculation. RVG9R:siSOD1 administration resulted in a substantial reduction of the SOD1 expression in the olfactory bulb $(63 \%)$, the cortex (47\%), the hippocampus $(61 \%)$, the thalamus $(53 \%)$, the hypothalamus $(55 \%)$, the midbrain $(39 \%)$, and the cerebellum (30\%) (Figure 4). In conclusion, the use of a positioning device enables an easy IN inoculation of RVG9R-complexed siRNA in mice, resulting in brain-specific siRNA delivery inducing functional silencing of the target gene. 

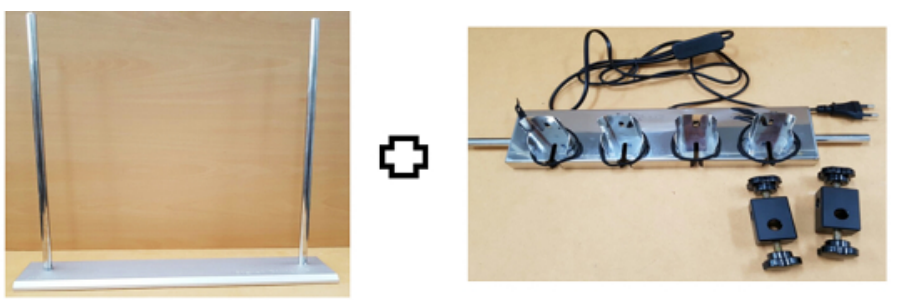

$\downarrow$ Assembly

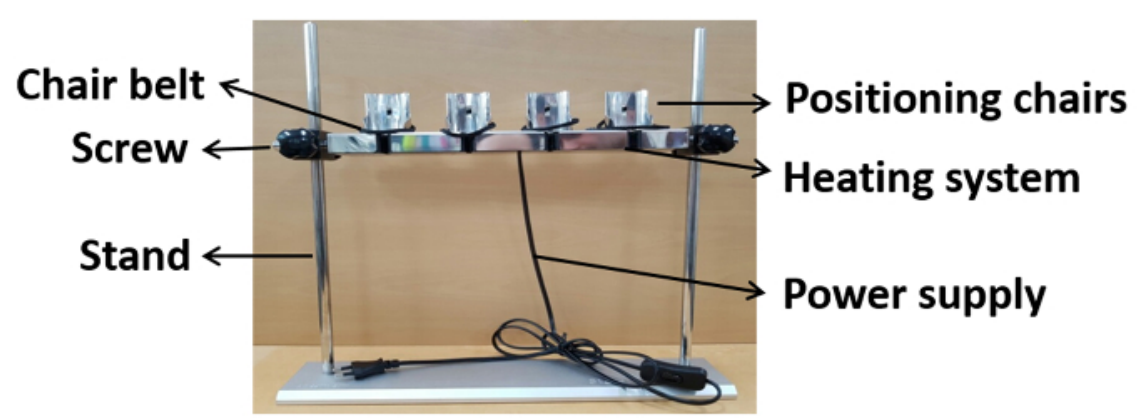

\section{Positioning device}

Figure 1: Photographic presentation of the device assembly. The positioning chairs are assembled on the stand at the appropriate height with screws. After the assembly, the device is connected to a power supply for heating to the physiological temperature during inoculation. Please click here to view a larger version of this figure. 


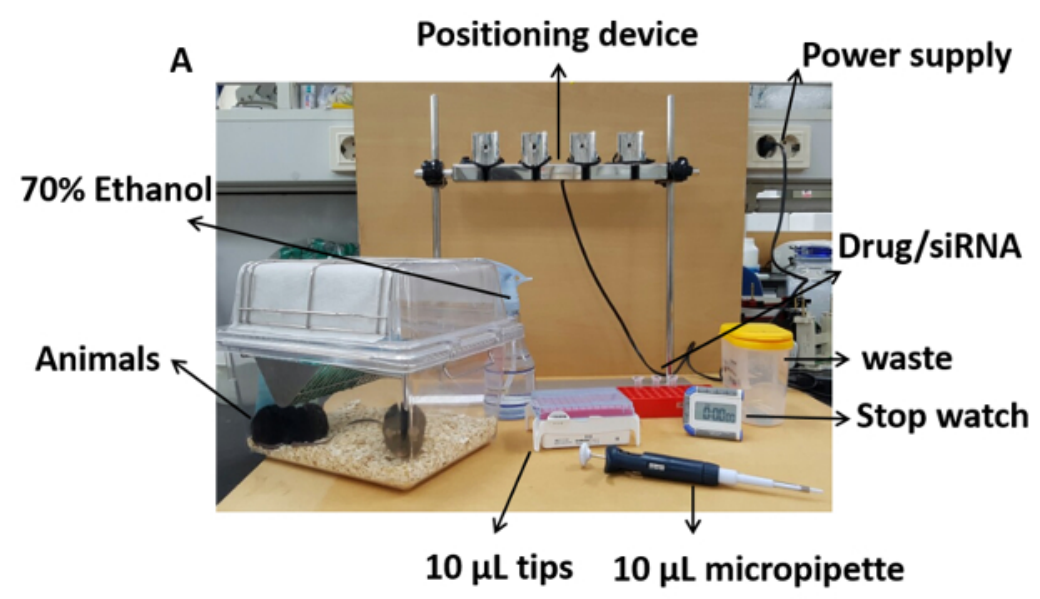

\section{B}

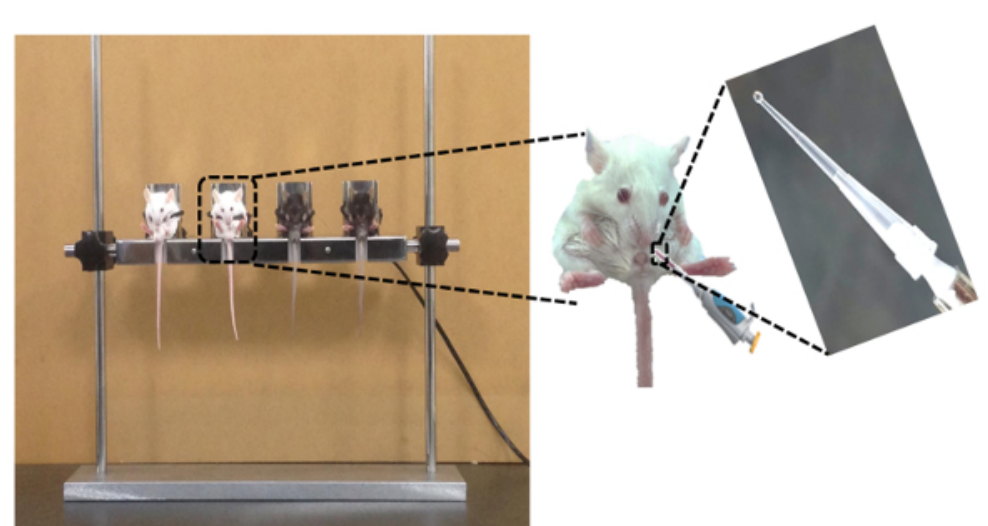

Figure 2: Photographic presentation of the IN inoculation procedure using the positioning device. (A) Experimental equipment required for the IN inoculation. (B) Animals in the head down-and-forward position seated on the device (left) and the deposition of a $2 \mu \mathrm{L}$ droplet for IN inoculation (right). Please click here to view a larger version of this figure. 




Figure 3: Distribution of fluorescently labeled peptide and siRNA after IN administration. (A) Biodistribution of intranasally inoculated RVG9R peptide at $48 \mathrm{~h}$ after a single IN inoculation. The brain and other peripheral organs were evaluated for the presence of fluorescence in mice inoculated with saline (PBS), $A^{488}, R V M-A^{488}$, and RVG- $A^{488}$ (left). Imaging of the mice treated as mentioned above while they are awake, in the supine position, and in the mouse positioning devise in the head down-and-forward position (right). (B) Biodistribution of the RVG9R:siRNA complex in the brain ( $n=3$ per group). The distribution of Cy5-labeled siRNA was visualized in full brain cryosections with a confocal laser microscope in animals inoculated with saline (PBS), siRNA complexed with control peptide (RVM9R-siCy5), or the brain-targeting peptide RVG9R (RVG9R-siCy5). The scale bar represents $1 \mathrm{~mm}$. Abbreviations: o.b = olfactory bulb; ctx = cortex; hippo = hippocampus; hypo = hypothalamus; tha $=$ thalamus; $m \cdot b=$ midbrain; cer = cerebellum. Please click here to view a larger version of this figure. 

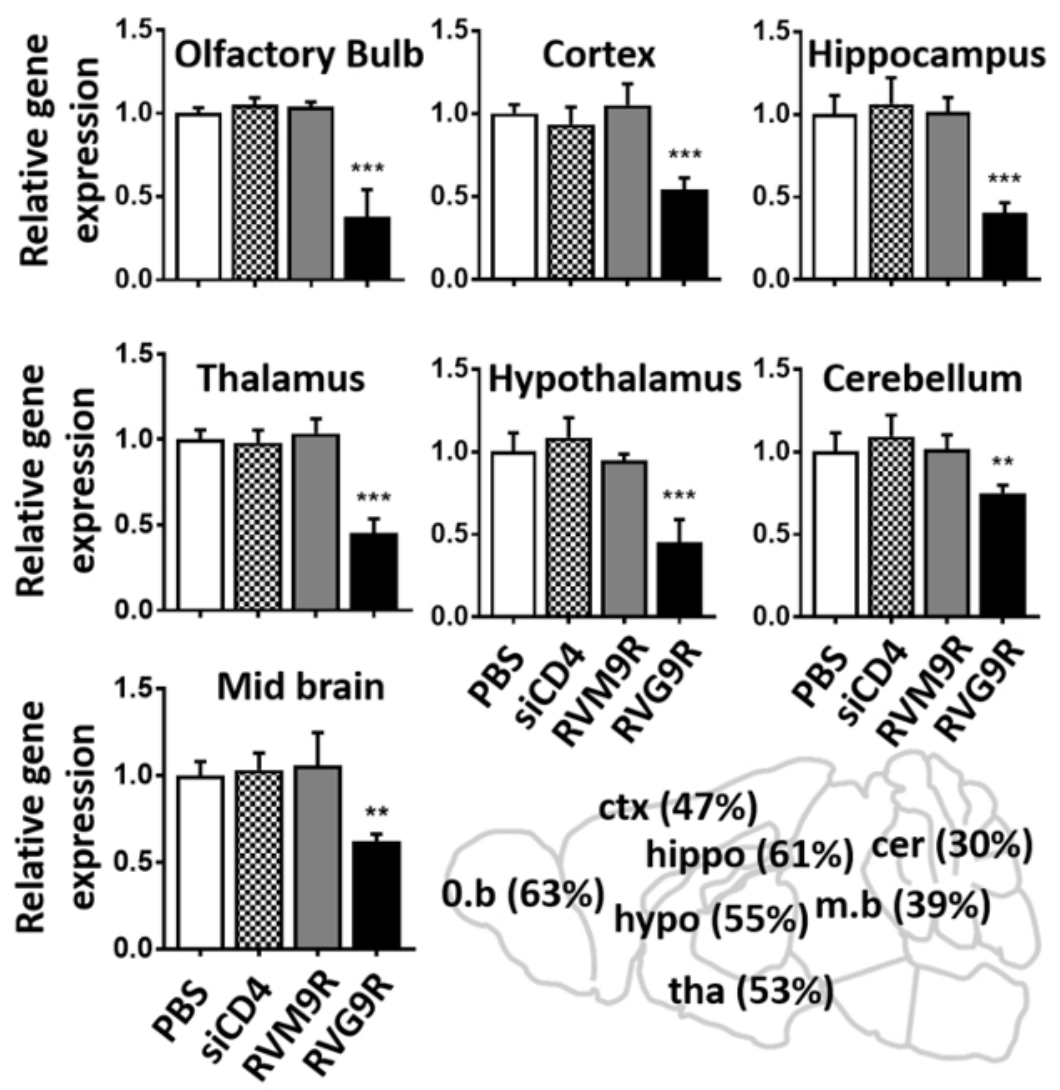

Figure 4: IN application of RVG9R:siSOD1 induces target gene silencing in multiple regions of the brain. qPCR analysis for murine SOD1 mRNA in indicated brain regions $24 \mathrm{~h}$ after the last IN inoculation of saline (PBS), RVG9R:siCD4 (siCD4), RVM9R:siSOD1 (RVM9R), and RVG9R:siSOD1 (RVG9R). The relative SOD1 silencing in the olfactory bulb, the cortex, the hippocampus (upper), the thalamus, the hypothalamus, the cerebellum (middle), and the midbrain (lower) is shown. Data represent the mean \pm SD relative to GAPDH after normalizing with the corresponding data of saline-treated mice $(n=3$ per group). The cartoon depicting the percentage of SOD1 mRNA in the indicated brain region after the IN inoculation is shown (lower right). ${ }^{* *} P<0.01,{ }^{* *} P<0.001$. Abbreviations: $0 . b=$ olfactory bulb; ctx $=$ cortex; hippo $=$ hippocampus; hypo = hippocampus; tha = thalamus; $m . b=$ midbrain; cer = cerebellum. Please click here to view a larger version of this figure.

\section{Discussion}

We have developed a mouse positioning device for optimally positioning mice for the nose-to-brain delivery of therapeutics. The device is equipped with different functionalities, ensuring the easy simultaneous handling of animals. It is also equipped with heating pads for the maintenance of the animals' physiological body temperatures during experimentation. The anesthetized mice can be maintained in the head down-and-forward position by means of specifically designed chairs, with minimal discomfort to the animals. The height of the positioning chairs can be adjusted in such a way as is best to visualize animal nostrils while administering drugs.

IN brain delivery has several intrinsic limitations, including the small surface area of nostrils (allowing maximum volumes of 20-30 $\mu \mathrm{L}$ per administration), nasal irritation, epithelium damage, and limited absorption across the nasal epithelium ${ }^{18}$. The IN administration of drugs to mice placed in the supine position has been used for brain delivery by dropping liquid drugs into the animals' alternate nares via pipette or polyurethane tubing $(24 \mathrm{G} \times 19 \mathrm{~mm})$ connected to microliter syringes ${ }^{19,20}$. Although the use of tubing systems to release drugs near the olfactory epithelium is a potentially suitable approach, it causes irritation or nasal inflammation upon repetitive administration. Moreover, the small size of the nasal cavity limits this approach, especially in mice that may be overcome by repeating, as well as by drug formulations that persist in the nasal mucosa. Another option is the IN administration of therapeutics to awake mice ${ }^{10}$. However, this approach requires skilled procedures for animal handling during IN inoculation. Additionally, it causes animal stress and, therefore, is not ideal for disease models, especially infectious disease models. Furthermore, inconsistent dosing may easily result from drug drainage into the lungs or the stomach due to imperfect animal gripping. The approach presented here allows scientists to overcome these technical barriers. The head down-and-forward position reduces the possibility of drug leakage from the nose to the lungs while inhaling, favoring direct and selective siRNA delivery to the brain. IN delivery using the mouse positioning device does not require any specialized technique for handling or gripping the animals during inoculation. Four animals at a time can be treated for a period of at least 30-45 min. The procedure can be scaled up by including an additional four-chair bar, allowing for the easy management of up to eight mice in the same experimental session. Therefore, following this method, a single operator may induce drug delivery to the brains of large groups of animals over extended periods of time.

The anatomical structure of the nasal cavity strongly influences the nose-to-brain delivery (as reviewed by Merkus et al. ${ }^{11}$ and Ruigrok and de Lang $\mathrm{e}^{21}$ ). The relative surface area of the nasal cavity in mice is 15 times bigger than that of humans and the relative surface area of the 
olfactory epithelium is 6 times bigger. Although there are significant differences in the anatomy of the nasal cavity in humans to rodents, there are approximately 45 clinical trials underway using the IN approach to treat several brain disorders (www.clinicaltrials.gov). The study presented here indicates that when using IN delivery of therapeutics, scientists should consider several factors, such as head position, sleeping, and proper delivery agents.

We have shown the efficient and specific deposition of fluorescently labeled siRNA to the mouse brain. Furthermore, the significant decrease in SOD1 gene expression observed after IN siRNA delivery confirmed functional effects. We previously showed consistently that the IN administration of RVG9R-siRNA complexes targeting the West Nile virus (WNV) RNA exerts a strong therapeutic effect on WNV encephalitis ${ }^{15}$. Notably, nose-to-brain siRNA delivery required a cell-targeting ligand (RVG) and a positively charged molecule (9R) to complex siRNA. In the absence of these elements, molecules were cleared through the systemic circuit and lymphatic vessels $48 \mathrm{~h}$ posttreatment (Figure $3 \mathrm{~A})^{15}$. Therefore, in the experimental setup described here, we have examined peptide/siRNA localization $48 \mathrm{~h}$ after inoculation to image only the levels retained specifically in the brain. This approach could be easily implemented for the delivery of other molecules, such as proteins, peptides, and nanoparticles, or other therapeutics, for the treatment of a number of brain-related disorders.

\section{Disclosures}

P.K. and S.K.L. are cofounders of Signet Biotech. P.K. is coinventor of patent PCT/US07/12,152, which partly includes claims relating to RVG9R. I.U., K.C., P.K., and S.K.L. are listed as coinventors of a submitted patent (PCT/KR2016/014220), which includes claims relating to a mouse positioning device.

\section{Acknowledgments}

This work was supported by the Korea Ministry of Health \& Welfare (HI17C1046) to S.K.L.

\section{References}

1. Pardridge, W. M. The blood-brain barrier: bottleneck in brain drug development. NeuroRx. 2, 3-14 (2005).

2. Glascock, J. J., et al. Delivery of therapeutic agents through intracerebroventricular (ICV) and intravenous (IV) injection in mice. Journal of Visualized Experiments. (56), e2968 (2011).

3. Khan, A. R., Liu, M., Khan, M. W., Zhai, G. Progress in brain targeting drug delivery system by nasal route. Journal of Controlled Release. 268, 364-389 (2017).

4. Meredith, M. E., Salameh, T. S., Banks, W. A. Intranasal delivery of proteins and peptides in the treatment of neurodegenerative diseases. The AAPS Journal. 17, 780-787 (2015).

5. Pardeshi, C. V., Belgamwar, V. S. Direct nose to brain drug delivery via integrated nerve pathways bypassing the blood-brain barrier: an excellent platform for brain targeting. Expert Opinion on Drug Delivery. 10, 957-972 (2013).

6. Agrawal, M., et al. Nose-to-brain drug delivery: An update on clinical challenges and progress towards approval of anti-Alzheimer drugs. Journal of Controlled Release. 281, 139-177 (2018)

7. Van Woensel, M., et al. Formulations for intranasal delivery of pharmacological agents to combat brain disease: a new opportunity to tackle GBM? Cancers. 5, 1020-1048 (2013).

8. Kulkarni, A. D., et al. Nanotechnology-mediated nose to brain drug delivery for Parkinson's disease: a mini review. Journal of Drug Targeting. 23, 775-788 (2015).

9. Dhuria, S. V., Hanson, L. R., Frey, W. H. Intranasal delivery to the central nervous system: mechanisms and experimental considerations. Journal of Pharmaceutical Sciences. 99, 1654-1673 (2010).

10. Hanson, L. R., Fine, J. M., Svitak, A. L., Faltesek, K. A. Intranasal administration of CNS therapeutics to awake mice. Journal of Visualized Experiments. (74), e4440 (2013).

11. Merkus, P., Ebbens, F. A., Muller, B., Fokkens, W. J. Influence of anatomy and head position on intranasal drug deposition. European Archives of Oto-Rhino-Laryngology and Head \& Neck. 263, 827-832 (2006).

12. Zeller, S., et al. Attachment of cell-binding ligands to arginine-rich cell-penetrating peptides enables cytosolic translocation of complexed siRNA. Chemistry \& Biology. 22, 50-62 (2015)

13. Kim, J., et al. Silencing CCR2 in macrophages alleviates adipose tissue inflammation and the associated metabolic syndrome in dietary obese mice. Molecular Therapy-Nucleic Acids. 5 (2016).

14. Kumar, P., et al. Transvascular delivery of small interfering RNA to the central nervous system. Nature. 448, 39 (2007).

15. Beloor, J., et al. Small interfering RNA-mediated control of virus replication in the CNS is therapeutic and enables natural immunity to West Nile virus. Cell Host \& Microbe. 23, 549-556.E3 (2018).

16. Ullah, I., et al. Trileucine residues in a ligand-CPP-based siRNA delivery platform improve endosomal escape of siRNA. Journal of Drug Targeting. 25, 320-329 (2017).

17. Yu, D., Li, G., Lesniak, M. S., Balyasnikova, I. V. Intranasal delivery of therapeutic stem cells to glioblastoma in a mouse model. Journal of Visualized Experiments. (124), e55845 (2017).

18. Mittal, D., et al. Insights into direct nose to brain delivery: current status and future perspective. Drug Delivery. 21, 75-86 (2014).

19. Renner, D. B., Frey II, W. H., Hanson, L. R. Intranasal delivery of siRNA to the olfactory bulbs of mice via the olfactory nerve pathway. Neuroscience Letters. 513, 193-197 (2012).

20. Serralheiro, A., Alves, G., Fortuna, A., Falcão, A. Intranasal administration of carbamazepine to mice: a direct delivery pathway for brain targeting. European Journal of Pharmaceutical Sciences. 60, 32-39 (2014).

21. Ruigrok, M. J., de Lange, E. C. Emerging insights for translational pharmacokinetic and pharmacokinetic-pharmacodynamic studies: towards prediction of nose-to-brain transport in humans. The AAPS Journal. 17, 493-505 (2015). 